\title{
Análisis de la Posesión de Balón en el Fútbol Juvenil en Relación a Variables de Situación: Estudio de Caso
}

\section{Analysis of the Ball Possession in Youth Soccer in Relation to Situational Variables: Case Study}

\section{Análise da Posse de Bola no Futebol Juvenil em Relação às Variáveis de Situação: Estudo de Caso}

\author{
Errekagorri, I.1, Castellano, J.1, Echeazarra, I.1 \\ ${ }_{1}$ Facultad de Educación y Deporte, Universidad del País Vasco (UPV/EHU), Vitoria-Gasteiz, España
}

\begin{abstract}
RESUMEN
El objetivo del presente estudio fue estudiar cómo afectaron en la posesión del balón de un equipo de fútbol perteneciente a la categoría División de Honor Juvenil de España durante una temporada competitiva las siguientes variables de situación: entrenador (entrenador 1, entrenador 2 y entrenador 3), nivel del oponente (alto, medio y bajo), ubicación del partido (local y visitante) y resultado final (ganar, empatar y perder). Se analizaron los 30 partidos disputados por el equipo de referencia. Para valorar el rendimiento del equipo se emplearon diferentes indicadores de rendimiento de posesión. Primero se realizó la prueba de Kruskal-Wallis para todas las variables y condiciones. Posteriormente solo en las variables estadísticamente significativas $(\mathrm{p}<0,05)$ se aplicó la prueba de $U$ de Mann-Whitney comparando las diferencias dos a dos. Los resultados mostraron en la variable nivel del oponente, que el equipo estuvo asociado con un descenso de la duración total del tiempo de posesión cuando se enfrentó con rivales de la zona alta de la clasificación a diferencia de cuando lo hizo con rivales de la zona media y baja. La principal conclusión del trabajo fue que la posesión de balón jugó un papel determinante en el rendimiento del equipo, si bien el nivel del oponente condicionó este indicador. Los entrenadores de fútbol formativo podrían incluir la posesión del balón como indicador de rendimiento, si se identifica con el modelo de juego, a partir de los cuales evaluar al equipo para proponer estrategias de intervención y optimizar su rendimiento.
\end{abstract}

Palabras clave: Fútbol formativo, deporte colectivo, Metodología Observacional, indicadores de rendimiento, variables contextuales.

\begin{abstract}
The aim of the present study was to study how the following situational variables affected the ball possession of a youth soccer team from Spain during a competitive season: coach (coach 1, coach 2 and coach 3), quality of opposition (high, medium and low), venue (home and away) and final outcome (win, draw and lose). The 30 league matches played by the reference team were analysed. Different possession performance indicators were used. First, the Kruskal-Wallis test was performed for all variables and conditions. Subsequently, only the Mann-Whitney U test was applied in the statistically significant variables $(p<0.05)$ comparing the differences two by two. The results showed in quality of opposition, that the team was associated with a decrease in the total length of time of possession
\end{abstract}

Correspondence to: Ibai Errekagorri Elezkano. Dirección Postal: Universidad del País Vasco (UPV/EHU). Facultad de Educación y Deporte (sección Deporte). C/ Portal de Lasarte. 01007 Vitoria-Gasteiz (España). Tel: 945013565. Email: ibai.errekagorri@ehu.eus 


\section{Errekagorri, Castellano, \& Echeazarra}

when faced with opponents from the high zone of the classification unlike when made with opponents from the medium and low zone. The main conclusion from the work was that the possession of the ball played a determining role in the performance of the team, although the quality of opposition conditioned this indicator. Soccer trainers of academies could include ball possession as a performance indicator, if it is identified with the game model, from which to evaluate the team to propose intervention strategies and optimize their performance.

Keywords: Soccer academy, team sport, Observational Methodology, performance indicators, contextual factors.

\section{RESUMO}

O objetivo do presente estudo foi estudar como as seguintes variáveis de situação afetaram a posse de um time de futebol juvenil na Espanha durante uma temporada competitiva: treinador (treinador 1, treinador 2 e treinador 3), nível do oponente (alto, médio e baixo), localização do jogo (casa e fora) e resultado final (vitória, empate e derrota). Os 30 jogos disputados pela time de referência foram analisadas. Para avaliar o desempenho da time, foram utilizados diferentes indicadores de desempenho. Primeiro, o teste de Kruskal-Wallis foi realizado para todas as variáveis e condições. Posteriormente, apenas o teste U de Mann-Whitney foi aplicado nas variáveis estatisticamente significantes $(\mathrm{p}<0,05)$ comparando as diferenças de dois a dois. Os resultados mostraram na variável nível do oponente, que a time esteve associada a uma diminuição no tempo total de posse quando confrontado com rivais da zona alta da classificação, diferentemente de quando feito com rivais na zona média e baixa. A principal conclusão do trabalho foi que a posse da bola desempenhou um papel determinante no desempenho da time, embora o nível do adversário condicionasse este indicador. Treinadores de futebol formativo poderiam incluir a posse da bola como um indicador de desempenho, se for identificado com o modelo de jogo, a partir do qual avaliar a equipe para propor estratégias de intervenção e otimizar seu desempenho.

Palavras chave: Futebol formativo, esporte coletivo, Metodologia Observacional, indicadores de desempenho, variáveis contextuais.

\section{INTRODUCCIÓN}

El análisis del rendimiento en el fútbol de élite supone un aspecto determinante para poder lograr el éxito deportivo (Lago-Peñas, Casáis, Domínguez, Martín, y Seirul-lo, 2010). Para tal fin, el uso de indicadores de rendimiento resulta imprescindible (Sarmento, Marcelino, Anguera, Campaniço, Matos, y Leitão, 2014). Uno de los indicadores de rendimiento que más atención ha tenido en los últimos años en el fútbol profesional ha sido la posesión de balón (Casal, Maneiro, Ardá, Marí, y Losada, 2017; Castellano, Casamichana, y Lago-Peñas, 2012). La elección de un estilo de juego es una decisión estratégica que el entrenador (o cuerpo técnico) toma en el intento de optimizar el rendimiento de los equipos que dirige (Fernández-Navarro, Fradua, Zubillaga, y McRobert, 2018). Gran parte de la literatura académica (Casal et al., 2017; Castellano, 2018; Jones, James, y Mellalieu, 2004; Lago-Peñas, Lago-Ballesteros, Dellal, y Gómez, 2010) coinciden en que la posesión de balón que caracteriza el modelo de juego indirecto, o basado en la posesión, acerca a los equipos al éxito, es decir, es un rasgo de los equipos ganadores y, por tanto, de los equipos que se sitúan en la zona alta de la tabla al finalizar la competición. Un aspecto importante cuando se analiza la posesión como un indicador de rendimiento es discernir la calidad de la misma, como aconseja Collet (2013). Por lo tanto, será necesario no solo cuantificar el tiempo que un equipo retiene la posesión, sino también contextualizarlo en la zona en la que se lleva a cabo (e.g., mantener el balón en zonas alejadas de la portería rival no garantiza el éxito de la fase ofensiva, aunque puede ser una estrategia recomendada para defender la posesión en circunstancias que lo recomiendan, Casal et al, 2017) o la eficacia de las mismas (e.g., ratio pases/tiros, Collet, 2013). No obstante, debido a que el fútbol es un deporte dominado por los factores tácticos y estratégicos, parece razonable considerar que las variables situacionales del juego pueden influir de alguna forma sobre el desempeño de los equipos (Lago-Peñas, Casáis, Domínguez, Lago-Ballesteros, y Rey, 2009). 


\section{Posesión y Variables de Situación en Fútbol Juvenil}

Algunas de las variables de situación que han sido estudiadas por los investigadores son: nivel del oponente (Aquino, Manechini, Bedo, Puggina, y Garganta, 2017; Gómez-Ruano, Mitrotasios, Armatas, y Lago-Peñas, 2018; Lago-Peñas, 2009; Lago-Peñas et al., 2010; Lago-Peñas, y Dellal, 2010; Lago-Peñas, y Lago-Ballesteros, 2011; Taylor, Mellalieu, James, y Shearer, 2008), jugar en casa o fuera (Aquino et al., 2017; Gómez-Ruano et al., 2018; Lago-Peñas, y Dellal, 2010; Lago-Peñas, y Lago-Ballesteros, 2011; Lago-Peñas, y Martín, 2007; Pollard, 2006; Sánchez, García-Calvo, Leo, Pollard, y Gómez, 2009), localización geográfica (Gutiérrez, Casamichana, Castellano, y Sánchez-Sánchez, 2018), horario (Gutiérrez, Castellano, Casamichana, y SánchezSánchez, 2018), resultado momentáneo (Casal et al., 2017; Lago-Peñas, 2009; Lago-Peñas et al., 2009; Lago-Peñas et al., 2010; Lago-Peñas, y Dellal, 2010), resultado final (Casal, Anguera, Maneiro, y Losada, 2019; Casal et al., 2017; Castellano, 2018; Collet, 2013; Lago-Peñas y Dellal, 2010), parte del partido (Castellano, Blanco-Villaseñor, y Álvarez, 2011) y tamaño del terreno de juego (González-Rodenas et al., 2019; Gutiérrez et al., 2018).

Una de las variables contextuales que más se ha analizado, por tener una influencia considerable en el rendimiento de los equipos (Lago-Peñas et al., 2009), es el nivel del oponente. Para analizar esta variable, los investigadores han optado por la división en niveles a partir de diferentes criterios. Por ejemplo, Taylor et al. (2008) propusieron una división simétrica basada en la clasificación final, fundamentando su análisis en la dicotomía "oposición fuerte" y "oposición débil". En otros estudios se diferenciaron tres (Castellano et al., 2011) o cuatro (Lago-Peñas, y Lago-Ballesteros, 2011) grupos, dependiendo de la posición final de los equipos en la clasificación de la liga. La mayoría de los trabajos (Aquino et al., 2017; Lago-Peñas, 2009; Lago-Peñas et al., 2010; Lago-Peñas, y Dellal, 2010) concluyen que jugar contra equipos de mayor nivel se asocia con una reducción en el tiempo de posesión del balón. Un estudio reciente (Fernández-Navarro et al., 2018) identificó que los equipos de fútbol en general juegan un estilo más directo, dejando a un lado el juego de posesión, cuando se enfrentan a rivales exitosos.

Otra variable contextual que parece condicionar de una forma trascendental el rendimiento de los equipos de fútbol es la ubicación del partido. Varios estudios (Aquino et al., 2017; Lago-Peñas, y Dellal, 2010;
Lago-Peñas, y Lago-Ballesteros, 2011; Lago-Peñas, y Martín, 2007; Pollard, 2006; Sánchez et al., 2009) han confirmado un resultado favorable en el mantenimiento de la posesión cuando los equipos juegan como locales en comparación a cuando lo hacen como visitantes: el ambiente local en el estadio, la familiaridad del entorno o el simple hecho de no tener que viajar, son algunas de las justificaciones que se dan (Pollard, 2006). Por su parte, Collet (2013) encontró que los equipos con mayor nivel que el resto dominan la posesión de balón independientemente de la ubicación del encuentro.

Otra variable de situación que cobra importancia en la investigación es el resultado final, asociándose un porcentaje alto de la posesión con la victoria en el encuentro (Lago-Peñas, y Dellal, 2010). En este sentido, Castellano (2018) expone en su estudio que la posesión de balón tiene una moderada correlación con los puntos obtenidos a lo largo de una temporada. En la misma línea, Casal et al. (2017) indicaron que en los partidos de la fase final de la UEFA EURO 2016 de Francia los equipos exitosos, es decir, los que obtuvieron la victoria, se caracterizaron por tiempos de posesión más largos, preferiblemente en la zona ofensiva media. Por su parte, Collet (2013) señala que la posesión parece tener un peso importante en la victoria, pero sugiere que eliminando de los análisis los equipos más exitosos, la posesión de balón no repercute de la misma manera en la acumulación de puntos por partido en las ligas nacionales.

Dentro de un mismo equipo, al margen de las adaptaciones contextuales, cambios relevantes en el seno de los clubes (e.g., cambio de entrenador) también podrían hacer variar el patrón de juego del equipo a lo largo de una misma temporada (Castellano, y Casamichana, 2016). La destitución del entrenador acontece cuando las expectativas del club no se están cumpliendo, en el intento de dar la vuelta a una mala dinámica de resultados u optimizar la cohesión del equipo (Balaguer, Castillo, Ródenas, Fabra, y Duda, 2014). Un trabajo previo (Castellano, y Casamichana, 2016) encontró diferencias significativas en cuanto al modelo de juego entre tres entrenadores que lideraron a un equipo durante una misma temporada. Los autores de este estudio, encontraron un descenso en la duración de la posesión de balón comparado el tercero de los entrenadores con los dos que le habían precedido. Sin embargo, cabe señalar, que el cambio de entrenador tiene un efecto positivo a corto plazo (e.g., cinco primeros partidos), permitiendo alcanzar a 


\section{Errekagorri, Castellano, \& Echeazarra}

los equipos un $20 \%$ más de puntos que en el mismo intervalo de tiempo por el entrenador cesado (LagoPeñas, 2007), si bien, este impacto desaparece a medio y largo plazo.

Tomando en cuenta todo lo anterior, el objetivo del estudio fue estudiar cómo afectaron algunas variables contextuales (entrenador, nivel del oponente, ubicación del partido y resultado final) en la posesión del balón de un equipo de fútbol de la categoría División de Honor Juvenil de España a lo largo de una temporada competitiva. Los resultados de este estudio permitirían a los entrenadores de fútbol que trabajan en la formación considerar la posesión del balón como indicador de rendimiento con el que evaluar a sus equipos $\mathrm{y}$, en su caso, proponer estrategias de intervención para optimizar su rendimiento.

\section{MATERIAL Y MÉTODOS}

\section{Diseño}

Dentro de los posibles diseños de observación aplicables en el ámbito de la actividad física y el deporte, y en relación a los tres criterios clave, unidades o participantes, temporalidad y dimensionalidad (Anguera, Blanco-Villaseñor, Hernández-Mendo, y Losada, 2011), el diseño del estudio está situado en el cuadrante I, atendiendo a los siguientes rasgos básicos: ideográfico, seguimiento y multidimensional. La ubicación en este cuadrante se justifica debido a que es una única unidad (equipo) que se observó, el registro se realizó durante toda la temporada para conocer la evolución del equipo y la codificación se llevó a cabo a partir de una herramienta de observación que aglutina varios criterios, dimensiones o macrocategorías (Bakeman, y Quera, 1996).

\section{Participantes}

En el presente estudio se analizaron los 30 partidos de liga disputados por un equipo de fútbol, del Grupo 2 correspondiente a la liga División de Honor Juvenil de España, perteneciente a las categorías inferiores de un club profesional militante de la Primera División Española de Fútbol (LaLiga) durante la temporada 2017-2018. De los 30 partidos de liga disputados por el equipo, 15 se jugaron en casa y 15 a domicilio. De todos estos partidos, 17 fueron victorias, siete empates y seis derrotas, terminando de esta manera en la zona alta de la clasificación a final de temporada. Todos los jugadores, padres-madres y/o tutores, así como los responsables del club fueron notificados del diseño de la investigación y sus requisitos, además de los beneficios y riesgos potenciales antes de iniciar el estudio. Todos firmaron el consentimiento informado. El Comité de Ética con Seres Humanos (CEISH) de la Universidad del País Vasco (UPV/EHU) aprobó el estudio.

\section{Herramienta de codificación}

Zonas del terreno de juego

El campo de fútbol se dividió transversalmente en cuatro zonas, que se designaron como Z1, Z2, Z3 y Z4. Esta forma de división está basada en la propuesta original de Castelo (1999) asociando a cada zona unos objetivos tácticos propios. Las zonas se diferenciaron por las propias líneas marcadas en el terreno de juego, tal y como queda reflejado en la Figura 1.

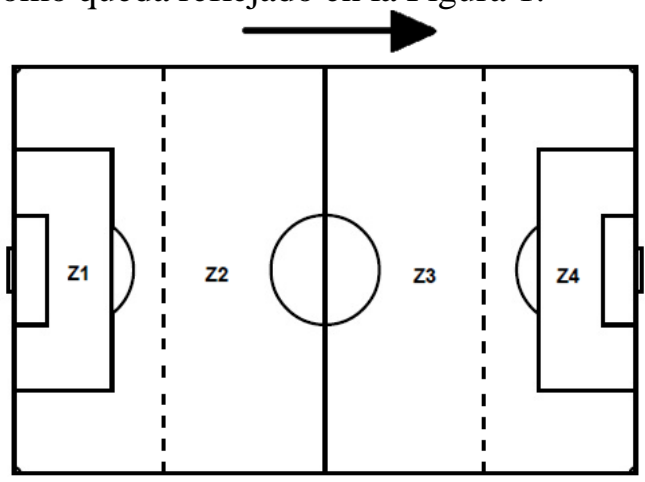

Figura 1. Subdivisión de espacios. El sentido de la flecha refleja el sentido del ataque del equipo de referencia.

\section{Indicadores de rendimiento}

En la Tabla 1 se recogen los códigos y las definiciones de los indicadores de rendimiento de posesión. Todos estos indicadores están relativizados al porcentaje de

\begin{tabular}{ll}
\multicolumn{2}{c}{ Tabla 1. Códigos y definiciones de indicadores de posesión. } \\
\hline Código & Definición \\
\hline POS\% & Porcentaje de la duración total de la posesión de balón. \\
POS1\% & Porcentaje de la duración de la posesión de balón en la Z1. \\
POS2\% & Porcentaje de la duración de la posesión de balón en la Z2. \\
POS3\% & Porcentaje de la duración de la posesión de balón en la Z3. \\
POS4\% & Porcentaje de la duración de la posesión de balón en la Z4. \\
\hline
\end{tabular}




\section{Posesión y Variables de Situación en Fútbol Juvenil}

la duración total del tiempo de juego efectivo del partido medido en minutos.

\section{Variables de situación}

$\mathrm{Al}$ igual que se ha propuesto en estudios anteriores (Aquino et al., 2017; Castellano, 2018; Castellano et al., 2011; Castellano, y Casamichana, 2016; Collet, 2013; Gómez et al., 2018; Lago-Peñas, 2009; LagoPeñas et al., 2010; Lago-Peñas, y Dellal, 2010; LagoPeñas, y Lago-Ballesteros, 2011; Lago-Peñas, y Martín, 2007; Pollard, 2006; Sánchez et al., 2009; Taylor et al., 2008), las variables de situación que se escogieron para este estudio fueron: entrenador, nivel del oponente, ubicación del partido y resultado final. Para la variable entrenador, los partidos disputados por el equipo de referencia (EQU) en la temporada 2017-2018 fueron agrupados dependiendo del entrenador que estuvo a su cargo en el momento de su análisis (Castellano, y Casamichana, 2016): entrenador 1 (E1) durante los primeros cinco partidos, entrenador 2 (E2) durante los segundos 11 partidos y entrenador 3 (E3) durante 14 partidos restantes hasta la jornada 30 .

En la variable nivel del oponente, los equipos fueron clasificados en tres niveles dependiendo de la clasificación al final del campeonato (Castellano et al., 2011): alto (OP1), medio (OP2) y bajo (OP3). De los 16 equipos del Grupo 2 correspondiente a la liga División de Honor Juvenil de España, los de nivel alto fueron los cinco primeros clasificados (incluyendo el EQU), los de nivel medio los siguientes seis clasificados y los de nivel bajo los últimos cinco clasificados.

En la variable ubicación del partido se añadieron los partidos jugados como local (L) y como visitante (V). De los 30 partidos de liga, 15 se jugaron en casa y 15 a domicilio. Dentro de la variable resultado final se incluyeron: ganar $(\mathrm{G})$, empatar $(\mathrm{E})$ y perder $(\mathrm{P})$. De los 30 partidos disputados por el EQU, 17 fueron victorias, siete empates y seis derrotas.

\section{Procedimiento}

Previo a la codificación definitiva de los partidos, se llevó a cabo la calidad del dato, a partir del estudio de la fiabilidad inter-observador. Para tal fin, dos observadores registraron in vivo la primera parte de un partido (fiabilidad inter) y tres meses después volvieron a realizar uno nuevo registro in vivo también (fiabilidad inter pero con el sentido de estabilidad). Una vez en disposición de los dos registros de ambas pruebas, se implementó el análisis de la concordancia entre observadores estimándose el coeficiente Kappa de Cohen (Cohen, 1988), utilizándose la aplicación informática GSEQ 5.1 para Windows (Bakeman, Quera, y Gnisci, 2009). Para dicho análisis se emplearon dos tipos de estrategia, a partir de considerar los registros como datos tipo evento o datos tipo estado. En la secuencia de eventos se considera únicamente la ocurrencia de las categorías, mientras que la secuencia de estados tiene en cuenta además la duración de cada una de las categorías (Anguera, Blanco-Villaseñor, Losada, y Hernández-Mendo, 2000). En la primera prueba, en ambos casos el valor de la Kappa fue óptimo, 0,91 y 0,70 para los eventos y los estados (con una ventana de tiempo de cinco segundos), respectivamente. En la segunda prueba, en ambos casos el valor de la Kappa también fue óptimo, 0,96 y 0,83 para los eventos y los estados (con una ventana de tiempo de cinco segundos), respectivamente. Dichos resultados pueden considerarse óptimos (Cohen, 1988) para llevar a cabo el estudio a partir de esta herramienta de codificación y con esta formación de los observadores.

Una vez superada la prueba de calidad y comprobada su estabilidad en el tiempo, todos los partidos codificados y registrados en EasyTag fueron exportados como archivos CSV a una hoja de cálculo de Microsoft Office Excel 2007 para su posterior análisis.

\section{Análisis estadístico}

Los datos son presentados con la media y la desviación estándar. Se emplearon pruebas no paramétricas para la comparación de medias de forma independiente entre las cuatro variables de clasificación definidas para la presente investigación: entrenador (E1, E2 y $\mathrm{E} 3)$, nivel del oponente (OP1, OP2 y $\mathrm{OP} 3)$, ubicación del partido (L y V) y resultado final (G, E y $\mathrm{P}$ ). Primero se realizó la prueba de Kruskal-Wallis para todas las variables y condiciones. Posteriormente solo en las variables estadísticamente significativas se aplicó la prueba de $U$ de Mann-Whitney comparando las diferencias dos a dos. Todos los análisis estadísticos fueron realizados utilizando el paquete estadístico SPSS v25.0 (SPSS Inc., Illinois, EE.UU.) para Windows y el nivel de significación admitido fue de $\mathrm{p}<0,05$. 


\section{Errekagorri, Castellano, \& Echeazarra}

\section{RESULTADOS}

En la Tabla 2 se muestran la media y la desviación estándar de los indicadores de rendimiento de posesión, diferenciándose cada una de las variables de clasificación estudiadas: entrenador, nivel del oponente, ubicación del partido y resultado final.

Según se recoge en la Tabla 2 existieron diferencias significativas $(p<0,05)$ entre E1 y E2 en POS3\%, siendo el EQU con el E1 su posesión estadísticamente superior. Con respecto a la variable de clasificación nivel del oponente (OP1, $\mathrm{OP} 2$ y $\mathrm{OP} 3)$, por una parte, existieron diferencias significativas $(\mathrm{p}<0,05)$ en POS\% entre OP1 y OP2, y por otra, entre OP1 y OP3, alcanzando valores superiores en OP2 y OP 3 respecto a OP1. Cabe destacar que también existieron diferencias significativas $(\mathrm{p}<0,05)$ entre OP1 y OP2 en POS1\%, siendo OP1 estadísticamente superior. También se pueden encontrar diferencias significativas $(\mathrm{p}<0,05)$ entre OP1 y OP2 en POS3\%, siendo en este caso OP2 estadísticamente superior. No se encontraron diferencias significativas entre el resto de indicadores. Finalmente, para las variables ubicación del partido (L y V) y resultado final (G, E y $P$ ) no existieron diferencias significativas en la posesión del EQU.

\section{DISCUSIÓN}

El propósito de este trabajo fue estudiar cómo afectaron en la posesión del balón de un equipo de fútbol perteneciente a la categoría División de Honor Juvenil de España durante una temporada competitiva las variables de situación: entrenador, nivel del oponente, ubicación del partido y resultado final. A conocimiento de los autores, éste es el primer trabajo en el que se aplican indicadores de posesión para el análisis del rendimiento ofensivo de un equipo de formación considerándose diferentes variables de situación, ya que este tipo de estudios han sido realizados en el fútbol profesional. Los principales resultados del estudio fueron que el equipo analizado mostró pequeñas diferencias relacionadas con los modelos de juego propuestos por los diferentes entrenadores que estuvieron a su cargo y, sobre todo, cuando el equipo se enfrentó a rivales de diferente calidad, establecida atendiendo a la posición alcanzada por los rivales al final del campeonato. $\mathrm{La}$ ubicación del partido y el resultado final, en cambio, no influyeron en la posesión del balón.
A partir de los resultados obtenidos, el equipo analizado tuvo más posesión que sus rivales durante el campeonato, si bien existieron diferencias en función de las variables de clasificación estudiadas. En cuanto a la variable de clasificación entrenador, cabe señalar que fue significativa entre E1 y E2 en el indicador POS3\%, siendo en E1 superior. Esto podría ser explicado por la prioridad del E1 de mantener la posesión en campo rival durante la fase de ataque en la búsqueda de la portería contraria y, posiblemente, haciendo que el bloque del equipo estuviera más adelantado. También Castellano y Casamichana (2016) encontraron diferencias respecto al peso de la posesión del balón como rasgo del modelo de juego de cada uno de los tres entrenadores de un mismo equipo durante una temporada, encontrándose que en 


\section{Posesión y Variables de Situación en Fútbol Juvenil}

Tabla 2. Porcentajes medios ( \pm desviación estándar) de los indicadores de rendimiento de posesión para cada variable de clasificación.

\begin{tabular}{|c|c|c|c|c|c|c|}
\hline \multirow[b]{2}{*}{$\begin{array}{l}\text { Variables de } \\
\text { clasificación }\end{array}$} & \multirow[b]{2}{*}{ Niveles } & \multicolumn{5}{|c|}{ Indicadores de rendimiento de posesión } \\
\hline & & POS $\%$ & POS $1 \%$ & POS $2 \%$ & $\begin{array}{c}\text { equipos de m } \\
\text { POS3\% }\end{array}$ & $\begin{array}{l}\text { or nivel con } \\
\text { POS } 4 \%\end{array}$ \\
\hline \multirow{3}{*}{ Entrenador } & $\mathrm{E} 1(\mathrm{~N}=5)$ & $56,5 \pm 7,4$ & $16,1 \pm 4,8$ & $32,7 \pm 7,5$ & $33,4 \pm 7,7^{b}$ & $17,9 \pm 4,8$ \\
\hline & $\mathrm{E} 2(\mathrm{~N}=11)$ & $56,9 \pm 8,2$ & $21,6 \pm 7,7$ & $36,4 \pm 8,7$ & $25,3 \pm 6,7$ & $16,7 \pm 6,2$ \\
\hline & E3 $(\mathrm{N}=14)$ & $58,6 \pm 6,9$ & $18,7 \pm 5,3$ & $37,2 \pm 5,2$ & $26,9 \pm 3,0$ & $17,3 \pm 3,4$ \\
\hline \multirow{3}{*}{ Oponente } & OP1 $(\mathrm{N}=8)$ & $48,6 \pm 5,1$ & $24,0 \pm 6,7^{b}$ & $34,4 \pm 5,3$ & $25,0 \pm 3,4$ & $41,6 \pm 6,7$ \\
\hline & $\mathrm{OP} 2(\mathrm{~N}=12)$ & $59,7 \pm 5,0^{\mathrm{a}}$ & $17,1 \pm 4,4$ & $34,7 \pm 6,3$ & $30,1 \pm 6,1^{\mathrm{a}}$ & $48,2 \pm 7,5$ \\
\hline & OP3 $(\mathrm{N}=10)$ & $62,4 \pm 4,1^{\mathrm{a}}$ & $18,3 \pm 6,7$ & $39,3 \pm 8,4$ & $26,0 \pm 6,7$ & $42,4 \pm 10,7$ \\
\hline \multirow{2}{*}{ Ubicación } & $\mathrm{L}(\mathrm{N}=15)$ & $58,7 \pm 7,3$ & $19,0 \pm 6,0$ & $34,8 \pm 6,5$ & $27,6 \pm 6,7$ & $18,6 \pm 4,1$ \\
\hline & $\mathrm{V}(\mathrm{N}=15)$ & $56,6 \pm 7,4$ & $19,6 \pm 6,9$ & $37,5 \pm 7,4$ & $27,1 \pm 5,3$ & $15,8 \pm 4,9$ \\
\hline \multirow{3}{*}{ Resultado } & $\overline{G(N=17)}$ & $58,3 \pm 7,1$ & $19,7 \pm 6,1$ & $35,6 \pm 7,8$ & $27,0 \pm 6,9$ & $17,7 \pm 5,1$ \\
\hline & $E(N=7)$ & $58,4 \pm 6,8$ & $17,3 \pm 4,8$ & $37,3 \pm 6,8$ & $27,8 \pm 3,6$ & $17,6 \pm 3,0$ \\
\hline & $P(N=6)$ & $54,8 \pm 8,9$ & $20,3 \pm 8,9$ & $36,5 \pm 5,2$ & $27,9 \pm 6,2$ & $15,2 \pm 5,2$ \\
\hline & Iedia $(\mathrm{N}=30)$ & $57,6 \pm 7,3$ & $19,3 \pm 6,3$ & $36,2 \pm 7,0$ & $27,4 \pm 6,0$ & $17,2 \pm 4,7$ \\
\hline
\end{tabular}

Nota: POS\% es el porcentaje de la posesión de balón del tiempo efectivo del partido, POS1\% es el porcentaje de la posesión de balón en la Z1 del tiempo en posesión de balón del equipo, POS2\% el porcentaje de la posesión de balón en la Z2, POS3\% el porcentaje de la posesión de balón en la Z3 y POS4\% el porcentaje de la posesión de balón en la Z4, E1 hace referencia a entrenador 1, E2 a entrenador 2 y E3 a entrenador 3, OP1 a nivel del oponente alto, OP2 a nivel del oponente medio, OP3 a nivel del oponente bajo, $\mathrm{L}$ a local, $\mathrm{V}$ a visitante, $\mathrm{G}$ a ganar, $\mathrm{E}$ a empatar y $\mathrm{P}$ a perder. b>E2, a $>\mathrm{OP} 1$ y c $>\mathrm{OP} 2$ para $\mathrm{p}<0,05$.

el periodo del tercer entrenador, el equipo mostró un descenso en la duración de la posesión de balón.

Con relación a la variable de clasificación nivel del oponente, cabe destacar que fue diferente la forma de jugar de la fase ofensiva dependiendo de la calidad de los rivales. Existieron diferencias significativas entre OP1 (el nivel más alto) y entre OP2 y OP3 en el indicador $\mathrm{POS} \%$, alcanzando valores superiores en OP2 y OP3 respecto a OP1, coincidiendo con otros estudios en el ámbito profesional (Aquino et al., 2017; Lago-Peñas, 2009; Lago-Peñas et al., 2010; LagoPeñas, y Dellal, 2010), que asocian el jugar contra reducción en el tiempo de posesión del balón. La variable de clasificación nivel del oponente también fue significativa entre OP1 y OP2 en el indicador POS $1 \%$, siendo superior en OP1. Este resultado puede explicarse por las dificultades que pudo encontrar el EQU para progresar a zonas más adelantadas contra equipos de mayor nivel desde su propio campo, concretamente desde la zona más cercana a su portería, por causa de la alta presión realizada por éstos. Es de extrañar que no se encontraran diferencias respecto a OP3, explicado quizás por el tipo de estrategia defensiva planteada por los equipos de menor nivel de presionar en bloque alto la salida de balón del EQU. Al igual que en los indicadores POS\% y POS $1 \%$, también fue significativa la diferencia entre OP1 y OP2 en el indicador POS3\%, siendo superior en OP2. Este resultado puede reflejar las dificultades que pudo tener el EQU de mantener la posesión en la Z3 del campo al enfrentarse a rivales de la parte alta de la clasificación, tal vez por la estrategia defensiva planteada por estos equipos de recuperar el balón cuanto antes en dicha zona e intentar generar un ataque 


\section{Errekagorri, Castellano, \& Echeazarra}

lo antes posible. Finalmente, cabe subrayar que no se encontraran diferencias respecto a OP3 en este último indicador. Esto puede ser explicado por el tipo de estrategia defensiva planteada por los equipos de menor nivel frente a un equipo clasificado entre los cinco primeros de la tabla como fue el equipo estudiado en el presente trabajo.

Referente a la variable de clasificación ubicación del partido, no se encontraron diferencias significativas a partir de los indicadores de posesión analizados. Estos resultados se ajustan con los de algunos autores (LagoPeñas, 2009; Lago-Peñas et al., 2010), que señalaron que no influía jugar en casa o a domicilio. Según Collet (2013), los equipos de mayor calidad habitualmente dominan la posesión de balón independientemente de que jueguen en casa o fuera. Esta afirmación puede ser una de las razones que explique la ausencia de diferencias, ya que el EQU terminó la temporada clasificado entre los cinco primeros.

Respecto a la variable resultado final, los resultados obtenidos no mostraron diferencias significativas. Éstos no se corresponden con los de otros estudios (Casal et al., 2019; Castellano, 2018; Collet, 2013), que relacionaron una mayor duración de la posesión de balón con la victoria en equipos profesionales. Quizá esta ausencia de asociación pudo deberse a la superioridad que mostró el EQU en su juego en la mayoría de los partidos disputados independientemente del resultado.

A pesar de que el presente estudio recoge la totalidad de los 30 partidos que el equipo disputó en una misma temporada competitiva, al tratarse de un estudio de caso, será necesario ser prudente a la hora de extrapolar estos resultados a otros equipos y temporadas. Creemos que la realización de estudio de un equipo en particular durante un período prolongado aporta información útil y sensible para el análisis del rendimiento en el fútbol de formación y deberían emplearse de manera adaptada a cada modelo de juego propuesto por los equipos, ya que el uso de datos agregados para varios equipos podría enmascarar los factores que determinan o contribuyen al éxito o fracaso de cada equipo en particular (Taylor et al., 2008). La segunda de las limitaciones del estudio podría concretarse en la ausencia de indicadores que informasen sobre el proceso defensivo o sin posesión del balón. La conexión entre la fase ofensiva y defensiva de los equipos es una cuestión crucial en el juego que hubiera permitido ofrecer una idea más global de cómo ha rendido el equipo en ambas facetas. CONCLUSIONES

A partir de los resultados del presente estudio se podría concluir que la posesión de balón jugó un papel importante en el rendimiento del EQU, puesto que finalizó la temporada entre los cinco primeros clasificados de la liga, teniendo más posesión que sus rivales. Además, apenas existieron diferencias en cuanto al modelo de juego propuesto por cada uno de los tres entrenadores, representado por la posesión del balón total y la realizada en diferentes zonas del campo. La presencia del rival jugó un papel importante afectando a los valores de posesión del balón del equipo estudiado, por lo que resulta inevitable la necesidad de contextualizar esta variable con relación al nivel del oponente.

\section{APLICACIONES PRÁCTICAS}

Los resultados obtenidos en este estudio podrían tener una doble aplicación práctica. En primer lugar, el seguimiento del rendimiento a partir del uso de indicadores de posesión podría ser de interés para el cuerpo técnico para evaluar si el modelo de juego propuesto por equipo se asocia a dinámicas positivas de rendimiento del equipo en competición, y en su caso, proponer estrategias de intervención con el objetivo de optimizarla, diseñando contenidos específicos en la semana de preparación previa, considerando la calidad del rival del próximo enfrentamiento. En segundo lugar, el seguimiento sobre el rendimiento del equipo con relación a la posesión de balón permitiría a la dirección deportiva valorar si el equipo está cumpliendo con las orientaciones marcadas desde el club sobre el modelo de juego que desea implantar en la academia.

\section{REFERENCIAS}

1. Anguera, M. T., Blanco-Villaseñor, Á., Hernández-Mendo, A., y Losada, J. L. (2011). Diseños observacionales: ajuste y aplicación en psicología del deporte. Cuadernos de Psicología del Deporte, 11(2), 63-76.

2. Anguera, M. T., Blanco-Villaseñor, Á., Losada, J. L., y Hernández-Mendo, A. (2000). La metodología observacional en el deporte: conceptos básicos. Lecturas: EF y Deportes. 


\section{Posesión y Variables de Situación en Fútbol Juvenil}

Revista Digital, 24, agosto 2000. http://www.efdeportes.com/efd24b/obs.htm [Consulta: 10 de abril de 2018].

3. Aquino, R., Manechini, J. P., Bedo, B. L. S., Puggina, E. F., y Garganta, J. (2017). Effects of match situational variables on possession: The case of England Premier League season 2015/16. Motriz. Revista de Educação Física, 23(3), e101794. https://doi.org/10.1590/s19806574201700030015

4. Bakeman, R., y Quera, V. (1996). Análisis de la Interacción: Análisis Secuencial con SDIS y GSEQ. Madrid: Ra-ma.

5. Bakeman, R., Quera, V., y Gnisci, A. (2009). Observer agreement for timed-event sequential data: A comparison of time-based and event-based algorithms. Behavior Research Methods, 41(1), 137-147. https://doi.org/10.3758/BRM.41.1.137

6. Balaguer, I., Castillo, I., Ródenas, L., Fabra, P., y Duda, J. L. (2015). Los entrenadores como promotores de la cohesión del equipo. Cuadernos de Psicología del Deporte, 15(1), 233-242. https://doi.org/10.4321/S157884232015000100022

7. Casal, C. A., Anguera, M. T., Maneiro, R., y Losada, J. L. (2019). Possession in Football: More Than a Quantitative Aspect - A Mixed Method Study. Frontiers in Psychology, 10, 501. https://doi.org/10.3389/fpsyg.2019.00501

8. Casal, C. A., Maneiro, R., Ardá, T., Marí, F. J., y Losada, J. L. (2017). Possession zone as a performance indicator in football. The Game of the Best Teams. Frontiers in Psychology, 8, 1176. https://doi.org/10.3389/fpsyg.2017.01176

9. Castellano, J. (2018). Relación entre indicadores de rendimiento y el éxito en el fútbol profesional. Revista Iberoamericana de Psicología del Ejercicio y el Deporte, 13(1), 4149.

10. Castellano, J., Blanco-Villaseñor, Á., y Álvarez, D. (2011). Contextual variables and time-motion analysis in soccer. International Journal of Sports $\begin{array}{lcc}\text { Medicine, } & 32(6), & 415-421 . \\ \text { https://doi.org/10.1055/s-0031-1271771 }\end{array}$

11. Castellano, J., y Casamichana, D. (2016). Mismos jugadores con diferentes entrenadores, ¿se puede jugar de manera diferente para optimizar el rendimiento en el fútbol profesional? SPORT TK: Revista Euroamericana de Ciencias del Deporte, 5(2), 133-140. https://doi.org/10.6018/264771

12. Castellano, J., Casamichana, D., y Lago-Peñas, C. (2012). The use of match statistics that discriminate between successful and unsuccessful soccer teams. Journal of Human Kinetics, 31, 139-147. https://doi.org/10.2478/v10078-0120015-7

13. Castelo, J. (1999). Fútbol. Estructura y dinámica del juego. Barcelona: INDE.

14. Cohen, J. (1988). Statistical power analysis: A computer program. Routledge.

15. Collet, C. (2013). The possession game? A comparative analysis of ball retention and team success in European and international football, 2007-2010. Journal of Sports Sciences, 31(2), 123-136.

https://doi.org/10.1080/02640414.2012.727455

16. Fernández-Navarro, J., Fradua, L., Zubillaga, A., y McRobert, A. P. (2018). Influence of contextual variables on styles of play in soccer. International Journal of Performance Analysis in Sport, 18(3), 423-436.

https://doi.org/10.1080/24748668.2018.1479925

17. Gómez-Ruano, M. A., Mitrotasios, M., Armatas, V., y Lago-Peñas, C. (2018). Analysis of playing styles according to team quality and match location in Greek professional soccer. International Journal of Performance Analysis in Sport, 18(6), 986-997. https://doi.org/10.1080/24748668.2018.1539382

18. González-Rodenas, J., Aranda-Malavés, R., Tudela-Desantes, A., Calabuig-Moreno, F., Casal, C. A., y Aranda, R. (2019). Effect of match location, team ranking, match status and tactical dimensions on the offensive performance in Spanish 'La Liga' soccer matches. Frontiers in 


\section{Errekagorri, Castellano, \& Echeazarra}

Psychology, 10,

2089.

https://doi.org/10.3389/fpsyg.2019.02089

19. Gutiérrez, J., Casamichana, D., Castellano, J., y Sánchez-Sánchez, J. (2018). Influencia de la localización geográfica de los partidos de fútbol en la respuesta física de equipos que compiten en la Segunda División Española. Journal of Sport and Health Research, 10(2), 295-302.

20. Gutiérrez, J., Castellano, J., Casamichana, D., y Sánchez-Sánchez, J. (2018). Influencia del tamaño del campo y horario del partido en la respuesta física de equipos de la Segunda División Española de Fútbol. Retos: nuevas tendencias en educación física, deporte y recreación, 33, 213216.

21. James, N., Mellalieu, S. D., y Hollely, C. (2002). Analysis of strategies in soccer as a function of European and domestic competition. International Journal of Performance Analysis in Sport, 2(1), 85-103. https://doi.org/10.1080/24748668.2002.1186826 3

22. Jones, P. D., James, N., y Mellalieu, S. D. (2004). Possession as a performance indicator in soccer. International Journal of Performance Analysis in Sport,4(1), 98-102. https://doi.org/10.1080/24748668.2004.1186829 5

23. Lago-Peñas, C. (2007). Aplicación de la regresión lineal en el estudio del impacto del cambio de entrenador sobre el rendimiento en el fútbol. Motricidad. European Journal of Human Movement, 19, 145-163.

24. Lago-Peñas, C. (2009). The influence of match location, quality of opposition, and match status on possession strategies in professional association football. Journal of Sports Sciences, 27(13), 1463-1469. https://doi.org/10.1080/02640410903131681

25. Lago-Peñas, C., Casáis, L., Domínguez, E., LagoBallesteros, J., y Rey, E. (2009). Influencia de las variables contextuales en el rendimiento físico en el fútbol de alto nivel. Motricidad. European
Journal of Human Movement, 23, 107-121. https://doi.org/10.5232/ricyde2009.01409

26. Lago-Peñas, C., Casáis, L., Domínguez, E., Martín, R., y Seirul-lo, F. (2010). La influencia de la localización del partido, el nivel del oponente y el marcador en la posesión del balón en el fútbol de alto nivel. Apunts. Educación Física y Deportes, 102, 78-86.

27. Lago-Peñas, C., y Dellal, A. (2010). Ball possession strategies in elite soccer according to the evolution of the match-score: the influence of situational variables. Journal of Human Kinetics, 25, 93-100. https://doi.org/10.2478/v10078-010-0036-z

28. Lago-Peñas, C., y Lago-Ballesteros, J. (2011). Game location and team quality effects on performance profiles in profesional soccer. Journal of Sports Science and Medicine, 10(3), 465-471.

29. Lago-Peñas, C., Lago-Ballesteros, J., Dellal, A., y Gómez, M. (2010). Game-related statistics that discriminated winning, drawing and losing teams from the Spanish soccer league. Journal of Sports Science and Medicine, 9(2), 288-293.

30. Lago-Peñas, C., y Martín, R. (2007). Determinants of possession of the ball in soccer. Journal of Sports Sciences, 25(9), 969974. https://doi.org/10.1080/02640410600944626

31. Pollard, R. (2006). Worldwide regional variations in home advantage in association football. Journal of Sports Sciences, 24(3), 231-240. https://doi.org/10.1080/02640410500141836

32. Sánchez, P., García-Calvo, T., Leo, F., Pollard, R., y Gómez, M. (2009). An analysis of home advantage in the top two Spanish Professional Football leagues. Perceptual and Motor Skills, 108(3), https://doi.org/10.2466/pms.108.3.789-797

33. Sarmento, H., Marcelino, R., Anguera, M. T., Campaniço, J., Matos, N., y Leitão, J. C. (2014). Match analysis in football: a systematic review. Journal of Sports Sciences, 32(20), 1831- 


\section{Posesión y Variables de Situación en Fútbol Juvenil}

1843.

https://doi.org/10.1080/02640414.2014.898852

34. Taylor, J., Mellalieu, S., James, N., y Shearer, D. (2008). The influence of match location, quality of opposition, and match status on technical performance in professional association football. Journal of Sports Sciences, 26(9), 885-895. https://doi.org/10.1080/02640414.2014.898852 